\title{
Disentangling the main populations of the Zodiacal Cloud from Zodiacal Light observations
}

\author{
R. Dumont ${ }^{1}$, J. B. Renard ${ }^{2}$, A. C. Levasseur-Regourd ${ }^{3}$, and J. L. Weinberg ${ }^{4}$ \\ ${ }^{1}$ Observatoire de Bordeaux, F-33270 Floirac, France \\ ${ }^{2}$ LPCE-CNRS, $3 A$ av. Recherche, F-45071 Orleans, France \\ ${ }^{3}$ Aéronomie CNRS, BP3, F-91371 Verrieres, France \\ ${ }^{4}$ MK Ind. LLC, 2137 E Flintstone Dr, Tucker, GA 30084, U.S.A.
}

(Received October 6, 1997; Revised January 31, 1998; Accepted February 21, 1998)

\begin{abstract}
Photometric surveys of the Zodiacal Light (ZL) already allowed to retrieve features of interplanetary dust space distribution and optical behaviour. Of the brightness "gathering" function $d Z=\mathfrak{D}(\alpha) / m$ along each line of sight (LOS), ( $\alpha$ being the phase angle, $m$ the subsolar distance of the LOS, $\mathfrak{D}$ the local scattering coefficient), two approximate values could be derived, based on the constraints provided by the two observed values of its integral $Z$, when the LOS is in-ecliptic. This "nodes of lesser uncertainty" method (Dumont, Levasseur-Regourd, Renard, 1985 to 1996), however, lowered but did not rule out question marks upon the phase function.

To improve this inversion, additional constraints can be found in ZL surveys from deep space probes. We show that both the Pioneer 10 (Toller and Weinberg, 1985) and (despite their lack of in-ecliptic scans) the Helios (Leinert et al., 1982) data imply the phase function to weakly depart from isotropy, at least in the $30^{\circ}-150^{\circ}$ range.

The latitudinal dependence $f(\beta \odot, r=\mathrm{cst})$ of the space density (less well known than the heliocentric, $\mathscr{D}(r, \beta \odot$ $=0)$ ) can be tracked through the brightness ratio, at the same elongation $\varepsilon$, aiming in the helioecliptic meridian, against in the ecliptic. At $\varepsilon=90^{\circ}$, this ratio 0.3 would lead-in the improper assumption of a single, homogeneous cloud - to fit the latitudinal density drop by $\mathrm{a} \simeq \cos ^{12} \beta \odot$ function. The resulting brightness ratio at $\varepsilon<90^{\circ}$, which should be equal to $\int_{\operatorname{LOS}} \cos ^{12} \beta_{\odot}(\alpha) \mathfrak{D}(\alpha) \cdot d \alpha / \int_{\operatorname{LOS}} \mathfrak{D}(\alpha) d \alpha$ turns out to be much lower than the ratio observed in the $60^{\circ}>\varepsilon>15^{\circ}$ range (again $\simeq 0.3$ ). This contradiction is solved with a steeper exponent (20-22?) for $\cos \beta \odot$, and by assuming the flattened cloud to coexist with another one, spherically symmetrical, which contributes $15-$ $25 \mathrm{~S} 10$ at $\varepsilon=90^{\circ}, 50-80 \mathrm{~S} 10$ at $\varepsilon=60^{\circ}, 100-160 \mathrm{~S} 10$ at $\varepsilon=45^{\circ}$ and $250-450 \mathrm{~S} 10$ at $\varepsilon=30^{\circ}$.
\end{abstract}

\section{Introduction}

Inverting the Zodiacal Light (ZL) brightness integral $Z$ in order to retrieve local values of the contribution $d Z$, hence of the space density and of the scattering function of the (elemental volume-averaged) interplanetary dust complex was a challenge for decades. Rigorous inversion is possible (Dumont, 1973) if the zodiacal cloud is assumed to be homogeneous (same properties everywhere, except density heliocentric-dependent by a power law $r^{-n}$ ), but this assumption turned out to be wrong when the Helios probes showed the polarisation degree (averaged along the line of sight-LOS) to change with spacecraft location.

In the further approach that we developed (Dumont and Levasseur-Regourd, 1985, 1988; Renard, 1992; Renard et al., 1995) the cloud was no longer regarded as homogeneous. The only assumptions were its cylindrical symmetry and a smoothness of the dust distribution, sufficient to regard the brightness "gathering" function along each LOS to also be smooth-say, fairly well represented with the help of 3 free parameters. Calling $\alpha$ the phase-angle and $\mathfrak{D}\left(\mathrm{AU}^{-1}\right)$ the local scattering coefficient, the local contribution $d Z$ to the

Copy right (C) The Society of Geomagnetism and Earth, Planetary and Space Sciences (SGEPSS); The Seismological Society of Japan; The Volcanological Society of Japan; The Geodetic Society of Japan; The Japanese Society for Planetary Sciences. brightness is $(S / m) \mathfrak{D}(\alpha) d \alpha$, where $S$ is the sun's intensity $\left(1.5 \cdot 10^{11}\right.$ if the brightness unit is the traditional S10) and $m$ is the subsolar distance of the LOS.

For a LOS in-ecliptic, two values of $Z$ are available from its intersections with earth's orbit (considered circular), which bring two constraints. The $3 \mathrm{~d}$ free parameter was relevant to the theoretically unknown phase function: varying it within its range of verisimilitude (i.e., rejecting every negative value of D) led us to point out the two regions of the LOS where all the "possible" D $(\alpha)$ curves do constrict. These "nodes of lesser uncertainty" turned out to be an efficient, although limited, tool for retrieving quantitative properties of the zodiacal cloud (heliocentric and latitudinal dependences of the local brightness, polarisation and-after comparison with IR observations - albedo and temperature).

\section{In-Ecliptic Scans and the Nearly Isotropic Scattering}

Only additional constraints, i.e. other values of $Z$ observed from $Z \neq 1 \mathrm{AU}$, can contribute to solve the remaining question: which is the real $\mathfrak{D}(\alpha)$ function? Deep space probes provide such data-although not profusely.

\subsection{Pioneer 10}

The ZL table by Toller and Weinberg (1985) gives brightnesses $Z$ in and near ecliptic, for eleven values of the 


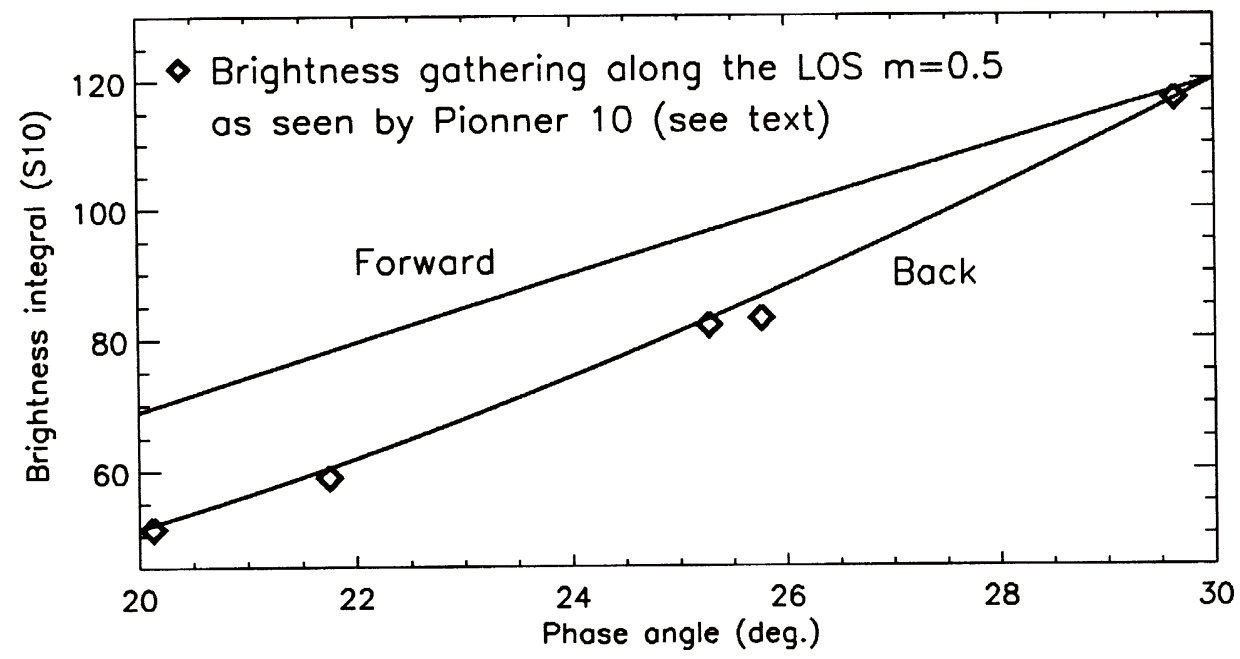

Fig. 1.

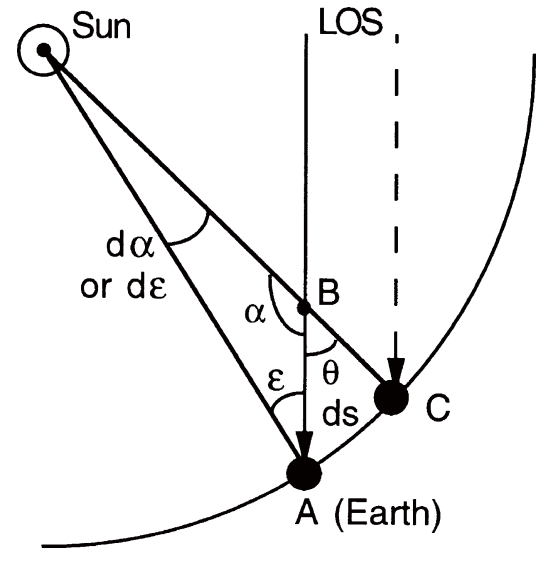

Fig. 2.

probe's heliocentric distance, $R$, and of the elongation, $\varepsilon$, by steps of $5^{\circ}$. Along a LOS relevant to earthbound observations ( $m \leq 1 \mathrm{AU})$, an interpolation through Toller and Weinberg's in-ecliptic figures into the elongations $\sin ^{-1}(m / R)$ leads to values of $Z$ along sections of the LOS exterior to earth's orbit-at least in the regions of the $(\mathrm{R}, \varepsilon)$ plane where $Z$ is bright enough ( $\geq 50 \mathrm{~S} 10)$ and the noise is low enough to allow a fairly safe interpolation. These conditions, and the empty boxes in the table, unfortunately restrict the sections of the LOS able to give valuable information. However, along the LOS at $m=0.5$ AU for instance, Fig. 1 shows how the brightness is seen by Pioneer 10 to be collected as a function of $\alpha$ in the 20.1 to $29.6^{\circ}$ range ( 51 to $117 \mathrm{~S} 10$ ). For comparison, the collecting slopes (normalized at $\alpha=30^{\circ}$ ) are shown for the two profiles $\mathfrak{D}(\alpha)$ considered in our paper of 1985 as limits for realistic departures from isotropic scattering, both in backscattering and in forwardscattering trends: $\mathfrak{D}\left(\alpha=150^{\circ}\right) / \mathfrak{D}\left(\alpha=30^{\circ}\right)=0.5$ to 6 , respectively. Pioneer 10 data therefore show that, in Fig. 3 of our 1985 paper, the quasi-symmetrical curve (quasi-isotropy with a slight backscattering trend) was much closer the truth than the other curve (which would mean a big forward effect). For other $m$ values, the result is the same.

\subsection{Helios}

Helios ZL results (Leinert et al., 1982) can provide other constraints inside earth's orbit. Although no LOS was available near the ecliptic, the very ubiquitous heliocentric dependence of the brightnesses at $\pm 16, \pm 31, \pm 90^{\circ}$ latitude (with a slight shift of the exponent from -2.25 at large elongations to -2.35 at small ones) seems to be safely extrapolable towards in-ecliptic LOS.

Figure 2 shows a LOS at elongation $\varepsilon$. The (infinitesimal) "final" section, BA, of the LOS is virtually isolated by introducing the subsequent location of the earth, $\mathrm{C}$, aligned with the sun and with $\mathrm{B}$. Let $d s$ be the length of the section BA. If its brightness contribution can be found, and how it depends upon elongation, i.e. upon scattering $(\theta)$ or phase $(\alpha)$ angles, then we can retrieve a range of the scattering or phase function (since, at A, $\theta=\pi-\alpha=\varepsilon$ ). We have:

$$
\begin{aligned}
d Z(\mathrm{BA}) & =Z(\mathrm{~A})-Z(\mathrm{~B}) \\
& =[Z(\mathrm{~A})-Z(\mathrm{C})]-[Z(\mathrm{~B})-Z(\mathrm{C})]
\end{aligned}
$$

where the differences in square brackets are both positive. The first difference is given by the observed elongation dependence of the ZL along the ecliptic, exactly by its derivative:

$$
[Z(\mathrm{~A})-Z(\mathrm{C})]=-(d Z / d \varepsilon) d \alpha=-Z^{\prime}(\varepsilon) d \alpha .
$$

It can be expressed proportionally to $d s$ ( sine law in triangle $\odot \mathrm{AB})$ and with $\varepsilon$ instead of $\alpha(d \varepsilon$ and $d \alpha$ having the same value):

$$
[Z(\mathrm{~A})-Z(\mathrm{C})]=-Z^{\prime}(\varepsilon) d \alpha=-Z^{\prime}(\varepsilon) \sin \varepsilon d s
$$

The second difference directly results from the abovementioned "allskyness" of the ZL gradients observed by Helios. Calling $r_{\mathrm{B}}$ the distance $\odot \mathrm{B}(\odot \mathrm{C}=1 \mathrm{AU})$, Helios power law at small elongations implies 


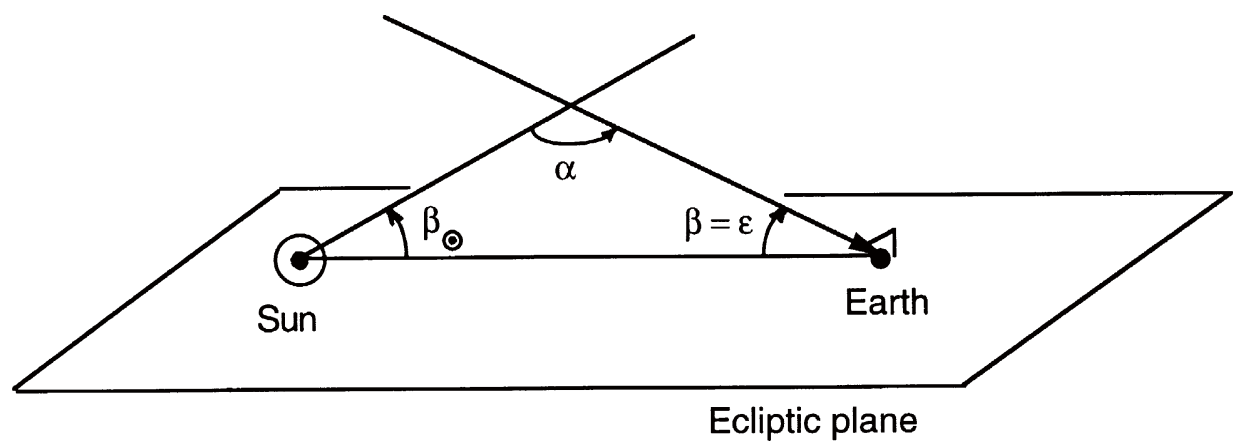

Fig. 3 .

Table 1

\begin{tabular}{|c|c|c|c|c|c|c|c|}
\hline \multirow[t]{2}{*}{$\varepsilon$} & \multicolumn{2}{|c|}{$Z_{\mathrm{M}} / Z_{\mathrm{E}}$} & \multicolumn{5}{|c|}{ Two clouds, values of $\Sigma(\mathrm{S} 10)$} \\
\hline & (obs.) & One cloud & $p=12$ & $p=17$ & $p=22$ & $p=27$ & $p=32$ \\
\hline $90^{\circ}$ & 0.31 & 0.30 & 0 & 15 & 22 & 27 & 31 \\
\hline $60^{\circ}$ & 0.30 & 0.21 & 44 & 58 & 67 & 73 & 77 \\
\hline $45^{\circ}$ & 0.30 & 0.17 & 110 & 130 & 140 & 150 & 160 \\
\hline $30^{\circ}$ & 0.26 & 0.13 & 290 & 330 & 350 & 370 & 390 \\
\hline
\end{tabular}

$$
Z(\mathrm{~B})=Z(\mathrm{C}) r_{\mathrm{B}}^{-2.35}
$$

so that

$$
[Z(\mathrm{~B})-Z(\mathrm{C})]=Z(\mathrm{C})\left[r_{\mathrm{B}}^{-2.35}-1\right]
$$

Then, triangle $\mathrm{ABC}$ gives $r_{\mathrm{B}} \cong 1-\cos \varepsilon d s$. Therefore,

$$
[Z(\mathrm{~B})-Z(\mathrm{C})] \cong 2.35 Z(\varepsilon) \cos \varepsilon d s
$$

(the approximations become rigorous formulae as $d s$ reaches zero). Subtracting Eq. (2) from Eq. (1) and dividing by $d s$, the expression for the angular dependence of the brightness contribution by the unit-length section at earth, i.e. for the scattering function of the interplanetary dust near terrestrial orbit is:

$$
\sigma(\theta=\varepsilon)=-\sin \varepsilon Z^{\prime}(\varepsilon)-2.35 \cos \varepsilon Z(\varepsilon) .
$$

To obtain $Z^{\prime}$, individual elongations are poorly reliable, contrary to a regression through the dots, if strongly correlated. Brightnesses at elongations 45, 40, 35 and $30^{\circ}$ obtained in our Tenerife campaign (Levasseur-Regourd and Dumont, 1980) and those at 30,21 and $15^{\circ}$ obtained by Leinert et al. (1976) in their rocket campaign (the latter normalized to Tenerife $\lambda, 502 \mathrm{~nm}$, after a weighted average of their $\lambda \lambda 468$, $475,591 \mathrm{~nm})$ are in excellent agreement at $30^{\circ}$ elongation (discrepancy: 3\%, still less at $475 \mathrm{~nm}$ ). A regression through these seven dots gives $\log Z=a-b \log \varepsilon(0.9991$ correlation coefficient). The phase function $\sigma(\theta=\varepsilon)=(b \sin \varepsilon / \varepsilon-$ $2.35 \cos \varepsilon) Z(\varepsilon)$ remains constant to $\pm 10 \%$ (320 S10.AU at $45^{\circ}, 381 \mathrm{~S} 10 . \mathrm{AU}$ at $15^{\circ}$ ). This implies a very slight forward- scattering enhancement. Although not exactly in the isotropic case, we consider fully justified to represent $\mathfrak{D}(\alpha)$ by functions "mirrored" at $90^{\circ}$ (an obvious consequence of isotropic scattering), all the more that a slight backscattering trend (which produces the gegenschein) acts over high scattering angles. Note that in a discussion (LevasseurRegourd and Dumont, 1978) of various observations at $\varepsilon=$ $45^{\circ}$ or less, an isotropic behaviour of the dust, or at most a very moderate forward effect, was already advocated.

\section{Out-of-Ecliptic Scans: Two Clouds Disentangled}

The brightness gathering function along a LOS in-ecliptic at elongation $\varepsilon$ is now reasonably well determined: Omitting the constant $(S / m)$, it is expressed by $Z_{\mathrm{E}}(\alpha)=\int_{0}^{\alpha} \mathfrak{D}(\alpha) d \alpha$, where the 3 constraints are provided by the abovementioned symmetry w.r.t. $90^{\circ}$, and by the 2 earthbound brightnesses at the intersections of the LOS with the orbit. In the helioecliptic meridian the brightness gathering at the same elongation is $Z_{\mathrm{M}}(\alpha)=\int_{0}^{\alpha} f\left(\beta_{\odot}(\alpha)\right) \mathfrak{D}(\alpha) d \alpha$, where $f\left(\beta_{\odot}\right)$ is the latitudinal dependence of the dust space density (see Leinert and Grün, 1990). Since $\beta_{\odot}=\pi-\alpha-\varepsilon$, the ratio $Z_{\mathrm{M}} /$ $Z_{\mathrm{E}}$ offers a good method of retrieving $f\left(\beta_{\odot}\right)$, for instance expressing it by $\cos ^{p} \beta \odot$, and adjusting the exponent $p$ to meet the observed ratio.

At $\varepsilon=90^{\circ}, Z_{\mathrm{M}} / Z_{\mathrm{E}}=0.3$ (Dumont and Sanchez (1976): 0.31; Levasseur-Regourd and Dumont (1980): 0.31; Frey et al. (1974): 0.30; Sparrow and Ney (1972): 0.30). In the old, obsolete view of a single, homogeneous cloud, this would have implied $p \simeq 12$. If we introduce the $Z_{\mathrm{M}} / Z_{\mathrm{E}}$ observed from Tenerife in the $60^{\circ} \geq \varepsilon \geq 30^{\circ}$ range, the table shows how the above assumption increasingly fails to meet them, as $\varepsilon$ decreases (column 3). Column 4 gives the brightnesses $\Sigma$ of a spherically symmetrical cloud, to be subtracted from both 
the $Z_{\mathrm{E}}$ and the $Z_{\mathrm{M}}$ if we want the $\left(Z_{\mathrm{M}}-\Sigma\right) /\left(Z_{\mathrm{E}}-\Sigma\right)$ to keep the ratios of column 3. Such a spherical component, however, cannot be expected to completely vanish at $\varepsilon=90^{\circ}$, but increasing $p$ allows to distort the $\Sigma(\varepsilon)$ dependence in order to keep a residual $\Sigma$ brightness at $\varepsilon=90^{\circ}$. Columns 5 to 8 show the brightnesses of our spherical cloud for $p$ increasing by steps of 5. Although an accurate determination of $p$ is of course out of reach, it seems highly noteworthy that:

1. A spherical cloud with density $\propto r^{-2}$ would be 16 times brighter at $30^{\circ}$ than at $90^{\circ}$ elongation-which agrees with the figures of column $6(p=22)$;

2 . No value of $p$ can fit the enhancement by 7 times, given between the same elongations by a cloud with density $\propto r^{-1}$.

Since Leinert's rocket data in the $30^{\circ} \geq \varepsilon \geq 15^{\circ}$ range gave $Z_{\mathrm{M}} / Z_{\mathrm{E}}=0.32$, they reinforce the likelihood of the spherical cloud, with $\Sigma$ contributions which could be as bright as 450 $\mathrm{S} 10$ at $30^{\circ}$ and $2000 \mathrm{~S} 10$ at $15^{\circ}$.

The value of $p$, however, has to decrease (18-20?) as well as the brightness $\Sigma$ of the spherical component $(\times 0.6$ to 0.8$)$ if the slight forward-scattering effect pointed out in the preceding section is assumed to be underestimated within the observational uncertainties.

Among the various models suggested for the zodiacal cloud, reviewed by Giese and Kneißel (1989) and Giese et al. (1986), those of the "sombrero" type were obvious precursors of the present dichotomic model. Instead of excessively distorting the unique cloud at high $\beta \odot$, however, the present view much more realistically introduces a second cloud with its own heliocentric and latitudinal characteristics.

\section{Conclusion}

In addition to the flattened, strictly speaking zodiacal cloud, heliocentric dependent about $r^{-1}$ and latitudinal dependent about $\cos ^{20} \beta_{\odot}$, the existence of a spherically symmetrical interplanetary cloud is invoked to account for the practically constant brightness ratio 0.3 between the two reference planes in the solar hemisphere. Although its heliocentric gradient could be steeper $\left(r^{-2} ?\right)$, this spherical cloud appears as a major component, since its brightness seems to exceed that of the flattened cloud at elongations less than $60^{\circ}$ when scanning the helioecliptic meridian.

\section{References}

Dumont, R., Phase function and polarization curve of interplanetary scatterers from zodiacal light photopolarimetry, Planet. Space Sci., 21, 2149-2155, 1973.

Dumont, R. and A. C. Levasseur-Regourd, Zodiacal Light gathered along the line of sight: Retrieval of the local scattering coefficient from photometric surveys of the ecliptic plane, Planet. Space Sci., 33, 1-9, 1985.

Dumont, R. and A. C. Levasseur-Regourd, Properties of interplanetary dust from infrared and optical observations: I. Temperature, global volume intensity, albedo and their heliocentric gradients, Astron. Astrophys., 191, 154-160, 1988.

Dumont, R. and F. Sanchez, Zodiacal Light photopolarimetry, III. Allsky survey from Teide 1964-1975 with emphasis on off-ecliptic features, Astron. Astrophys., 51, 393-399, 1976.

Frey, A., W. Hofmann, D. Lemke, and C. Thum, Photometry of the Zodiacal Light with the Balloon-borne telescope THISBE, Astron. Astrophys., 36, 447-454, 1974.

Giese, R. H. and B. Kneißel, Three-dimensional models of the zodiacal dust cloud, II. Compatibility of proposed infrared models, Icarus, 81, 369-378, 1989.

Giese, R. H., B. Kneißel, and U. Rittich, Three-dimensional models of the zodiacal dust cloud, a comparative study, Icarus, 68, 395-411, 1986.

Leinert, Ch. and E. Grün, Interplanetary dust, in Physics of the Inner Heliosphere I, edited by R. Schwenn and E. Marsch, pp. 207-275, 1990.

Leinert, Ch., H. Link, E. Pitz, and R. H. Giese, Interpretation of a rocket photometry of the inner Zodiacal Light, Astron. Astrophys., 47, 221230, 1976.

Leinert, Ch., I. Richter, E. Pitz, and M. Hanner, HELIOS Zodiacal Light measurements-a tabulated summary, Astron. Astrophys., 110, 355357, 1982.

Levasseur-Regourd, A. C. and R. Dumont, Sur l'isotropie de la fonction de phase dans la diffusion par les particules interplanétaires, C.R.A.S. Paris, 286, B-61-B-64, 1978.

Levasseur-Regourd, A. C. and R. Dumont, Absolute photometry of the Zodiacal Light, Astron. Astrophys., 84, 277-279, 1980.

Renard, J. B., Mise en évidence de l'évolution des propriétés optiques et physiques des grains dans le système solaire, Thesis, Univ. Paris VI, 1992.

Renard, J. B., A. C. Levasseur-Regourd, and R. Dumont, Properties of interplanetary dust from infrared and optical observations, II. Brightness, polarization, temperature, albedo and their dependence on the elevation above the ecliptic, Astron. Astrophys., 304, 602-608, 1995.

Sparrow, J. G. and E. P. Ney, Observations of the Zodiacal Light from the ecliptic to the poles, Astrophys. J., 174, 705-716, 1972.

Toller, G. N. and J. L. Weinberg, The change in near-ecliptic Zodiacal Light brightness with heliocentric distance, in $A S S L, 119$, edited by $\mathrm{R}$. H. Giese and Ph. Lamy, pp. 21-25, 1985. 